\title{
Some Connections between Minkowski and Hyperbolic Planes
}

\author{
by \\ Jarosław KOSIOREK and Andrzej MATRAŚ \\ Presented by Janusz RYCHLEWSKI
}

Dedicated to Walter Benz

Summary. The model of the Minkowski plane in the projective plane with a fixed conic sheds a new light on the connection between the Minkowski and hyperbolic geometries. The construction of the Minkowski plane in a hyperbolic plane over a Euclidean field is given. It is also proved that the geometry in an orthogonal bundle of circles is hyperbolic in a natural way.

Introduction. Using ideas of H. Beck (cf. [2]) W. Benz observed the possibility to represent circles of the incidence Minkowski plane over the reals as the sets of oriented lines tangent to horocycles, equidistant curves and circles of hyperbolic geometry (cf. [3], [5]). Using the projective model of Minkowski geometry (cf. [8]) we prove that we get in fact the model of the incidence Minkowski plane in a hyperbolic plane over any Euclidean field. This result is analogous to the connection between Laguerre and Euclidean geometry (cf. [10]). In Section 3 we establish that hyperbolic geometry occurs in a bundle of circles of the symmetric Minkowski plane.

1. Notations and basic definitions. Let $C$ be a fixed proper conic in a Fano-Pappus projective plane $\Pi$ and $\mathbf{P G L}(C)$ be the group of projectivities of this conic. This group is isomorphic to the group $\mathbf{P G L}(2, F)$ for some commutative field $F$ with char $F \neq 2$. According to connections between symmetric Minkowski planes (cf. [6]) and PGL(2,F) (cf. [7]) we obtain (cf. [8])

2000 Mathematics Subject Classification: Primary 51B20.

Key words and phrases: symmetric Minkowski plane, hyperbolic plane, projectivity on a conic. 
TheOREM 1.1. Every symmetric Minkowski plane $\mathcal{M}$ with char $\mathcal{M} \neq 2$ is isomorphic to the structure $\left(\mathcal{P}^{C}, \Lambda^{C}, \Sigma_{1}^{C}, \Sigma_{2}^{C}, \in\right)$, where

$$
\begin{aligned}
& \mathcal{P}^{C}=\{(x, y) \mid x, y \in C\}, \\
& \Lambda^{C}=\{\{(x, y) \mid x, y \in C, y=\alpha(x)\} \mid \alpha \in \mathbf{P G L}(C)\}, \\
& \left.\Sigma_{1}^{C}=\{\{(x, y) \mid x, y \in C, x=a\} \mid a \in C\}\right\}, \\
& \left.\Sigma_{2}^{C}=\{\{(x, y) \mid x, y \in C, y=b\} \mid b \in C\}\right\},
\end{aligned}
$$

and $C$ is an arbitrary fixed proper conic.

In analogy with the projective model of hyperbolic geometry, we will call the structure from Theorem 1.1 the projective model of Minkowski geometry. The element of the set $\Lambda^{C}$ corresponding to a circle determined by the projectivity $\alpha$ of $C$ will be denoted by $K_{\alpha}$. The center and axis of the projectivity $\alpha$ will be denoted by $o_{\alpha}, O_{\alpha}$ resp. (cf. [11]). The circle $K_{\alpha}$ is orthogonal to $K_{\text {id }}$ in this model (written $K_{\alpha} \perp K_{\text {id }}$ ) when $\alpha$ is an involution. For any involution $\alpha$ and projectivity $\beta \neq$ id we have $K_{\alpha} \perp K_{\beta} \Leftrightarrow o_{\alpha} \in O_{\beta}$ (cf. [8]).

Corollary 1.1. For every circle $E$ of the symmetric Minkowski plane $\mathcal{M}$ with char $\mathcal{M} \neq 2$ there exists an isomorphism between $\mathcal{M}$ and its projective model such that $K_{\mathrm{id}}$ corresponds to the circle $E$.

The isomorphism described in Corollary 1.1 will be denoted by $\Phi_{\mathcal{M}}^{E}$. The pencil of circles orthogonal to two different circles $K, L$ will be denoted by $(K, L)^{\perp}$, and the bundle of circles orthogonal to the circle $K$ will be denoted by $K^{\perp}$ (cf. [7], [12]). We will use the following results from [8]:

TheOREM 1.2. For any triple of pairwise orthogonal circles $K, L, M$ of the symmetric Minkowski plane with char $\mathcal{M} \neq 2$ there exists exactly one circle $N$ such that $N \perp K, L, M$.

Proposition 1.1. If $M \neq N$ and $M, N \in(K, L)^{\perp}$ then $(K, L)^{\perp}=$ $(M, N)^{\perp}$, i.e. the pencil of circles is determined by two different circles.

Proposition 1.2. Each nonparabolic pencil is determined by a pair of orthogonal circles of this pencil, one of which can be chosen arbitrarily.

Proposition 1.3. In the projective model of Minkowski geometry the pencil conjugate to $\left(K_{\mathrm{id}}, K_{\alpha}\right)^{\perp}$ is the set $\left\{K_{\beta} \mid O_{\beta}=O_{\alpha}\right\} \cup\left\{K_{\mathrm{id}}\right\}$.

Let $\Pi=(\mathcal{P}, \mathcal{L}, \in)$ be the Fano-Pappus projective plane and $C$ be a fixed conic. An extended hyperbolic plane is the structure $\overline{\mathbf{H}}=\left(\mathcal{P}, \mathcal{L}, \in, \perp_{h}\right)$ where $A \perp_{h} B$ when $A$ and $B$ are conjugate with respect to $C$ ([4], [9]). When the plane is over a Euclidean field we get the classical Klein model $\mathbf{H}=\left(\mathcal{U}, \mathcal{L}_{U}, \in, \perp_{U}\right)$ of hyperbolic geometry, where $\mathcal{U}$ is the interior of the conic, $\mathcal{L}_{U}=\{\mathcal{U} \cap L \mid L \in \mathcal{L} \wedge L \cap \mathcal{U} \neq \emptyset\}, \mathcal{U} \cap A \perp_{U} \mathcal{U} \cap B \Leftrightarrow A \perp_{h} B$. 
Every structure isomorphic to this model will be called a hyperbolic plane. For a comprehensive axiomatic exposition of such hyperbolic geometry we refer the reader to [1].

2. The hyperbolic model of Minkowski geometry. The projective model of Minkowski geometry and its geometric interpretation indicate the possibility to construct a model of Minkowski plane in classical hyperbolic geometry (cf. [3]). Let us consider classical hyperbolic geometry such that every line contains exactly two ideal points and its Klein model $\mathbf{H}=\left(\mathcal{U}, \mathcal{L}_{U}, \in, \perp_{U}\right)$ in the projective plane over a Euclidean field. Because every line contains two different ideal points, we can define an orientation of the line by singling out one of the ideal points. This means that in the Klein model an oriented line is an ordered pair of points of $C$.

As the set $\mathcal{P}^{H}$ of points of the hyperbolic model of Minkowski geometry we take the oriented lines of the hyperbolic plane and the ideal points. In the Klein model they correspond to oriented pairs $(x, y)$ of points of $C$ and points of $C$ (pairs $(x, x), x \in C$ ).

To define the set of lines we use the following lemma:

Lemma 2.1. For every projectivity $\alpha$ of $C$ such that $\alpha(x)=x^{\prime} \neq x$, $\alpha(y)=y^{\prime} \neq y$ there exist involutions $\beta, \gamma$ of $C$ with $o_{\beta}, o_{\gamma} \in O_{\alpha}, \gamma \beta(x)=$ $x^{\prime}, \gamma \beta(y)=y^{\prime}$.

Proof. We define $o_{\beta}=x x^{\prime} \cap O_{\alpha}$ and $o_{\gamma}=x y^{\prime} \cap x^{\prime} y$. By definition of axis, $o_{\gamma} \in O_{\alpha}$. We obtain $\gamma \beta(x)=\gamma\left(x^{\prime}\right)=y, \gamma \beta\left(x^{\prime}\right)=\gamma(x)=y^{\prime}$.

In classical hyperbolic geometry a circle, a horocycle and an equidistant curve are defined as orbits of points with respect to the group of displacements generated by symmetries with axes of a proper pencil, parallel line pencil and ultraparallel line pencil, respectively. More exactly in the last case we obtain one segment of an equidistant curve and to get the complete curve one has to add to the set of generators of the group the symmetry with respect to the common perpendicular. In the Klein model these are conics with appropriate properties. According to the definition of points of the projective model of the Minkowski plane we introduce the following convention.

A circle (resp. horocycle) of oriented lines is defined to be the orbit of an oriented line with respect to the subgroup of the proper isometries of the group generated by symmetries with axes belonging to a proper pencil (resp. pencil of parallel lines). An equidistant curve of oriented lines is the union of the orbit of an oriented line with respect to the subgroup of proper isometries of the group generated by symmetries with axes belonging to a pencil of ultraparallel lines and their symmetric image with respect to the axes of the pencil. 
We remark that in the case of a circle and an equidistant curve we allow the case in which the oriented line is incident with the center of the pencil (resp. is perpendicular to the axis of the pencil). Such circles and equidistant curves are called degenerate in analogy to the classical picture, in which the circle degenerates to a point and the equidistant curve to a line.

The set of circles of the hyperbolic model of Minkowski geometry, denoted by $\Lambda^{H}$, consists of the following sets of points:

(i) the set of all ideal points,

(ii) circles of oriented lines,

(iii) horocycles of oriented lines with ideal points corresponding to pencils,

(iv) equidistant curves of lines with ideal points of the axes of corresponding pencils.
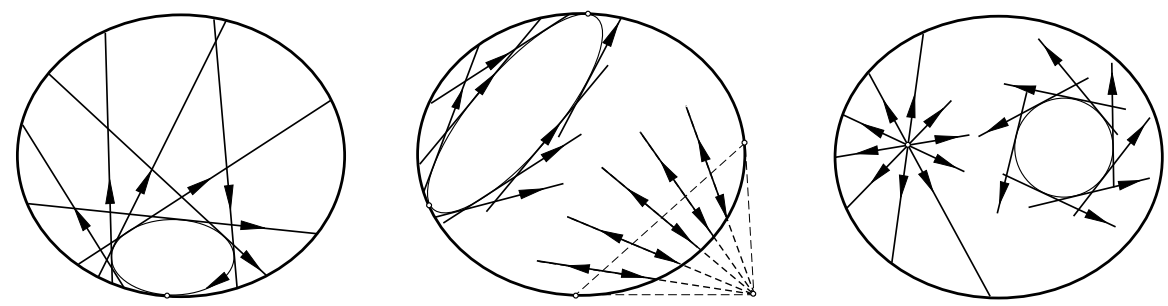

Fig. 1. Circles of the hyperbolic model of the Minkowski plane illustrated in the Klein model

(i) Ideal points of a hyperbolic plane correspond to points of $C$ in the Klein model, i.e. the circle $K_{\text {id }}$ of the projective model.

(ii) Let $K_{\alpha}$ be an arbitrary circle of the projective model of Minkowski geometry such that $\alpha$ has no fixed points. The projectivity $\alpha$ is uniquely determined by its axis $O_{\alpha}$ and the image $x^{\prime}=\alpha(x)$ of one point, so the center $o_{\alpha}$ is a proper point because $O_{\alpha}$ is disjoint from $C$. By Lemma 2.1 for any other pair $y, y^{\prime}=\alpha(y)$ there exist involutions $\beta, \gamma$ such that $\gamma \beta(x)=$ $y, \gamma \beta\left(x^{\prime}\right)=y^{\prime}$ and $o_{\alpha} \in O_{\beta} \cap O_{\gamma}$. In the Klein model that means that every oriented line determined by the pair $\left(y, y^{\prime}\right)$ is the image of an oriented line represented by the pair $\left(x, x^{\prime}\right)$ under an isometry which is a superposition of two symmetries with axes through the point $o_{\alpha}$. Hence the circle $K_{\alpha}$ corresponds to a hyperbolic circle of oriented lines.

(iii) If the projectivity is parabolic, then $O_{\alpha}$ is tangent to $C$ with the point of contact $o_{\alpha}$, which is the unique fixed point of $\alpha$. The other points of $O_{\alpha}$ are outside $C$. Analogously to (ii) we see that to $K_{\alpha}$ there corresponds a horocycle of oriented lines with ideal points of a suitable pencil which is associated to the point $\left(o_{\alpha}, o_{\alpha}\right)$ of $K_{\alpha}$. 
(iv) The axis $O_{\alpha}$ contains points inside $C$ because it intersects $C$ in two different points. Involutions from Lemma 2.1 are not necessarily axial symmetries. One or both of them are symmetries with respect to a point (when $o_{\beta}$ or $o_{\gamma}$ is inside $C$ ). It is known that such a symmetry is a superposition of axial symmetries: one with axis $O_{\alpha}$ and another with an axis through $o_{\alpha}$. It follows that to $K_{\alpha}$ corresponds an equidistant curve of oriented lines with ideal points belonging to the axis of the relevant pencil, so the ideal points are $(p, p)$ and $(q, q)$ where $p, q$ are fixed points of $\alpha$.

As a class of generators $\Sigma_{1}^{H}$ of the hyperbolic model of Minkowski geometry we take pencils of parallel lines oriented from their common ideal point to the ideal point. The second class of generators $\Sigma_{2}^{H}$ consists of pencils of parallel lines oriented from their specific ideal point to the common ideal point. Of course such generators uniquely correspond to generators of the projective model of Minkowski geometry.

Summarizing, the above remarks give

TheOREM 2.1. The hyperbolic model $\left\langle\mathcal{P}^{H}, \Lambda^{H}, \Sigma_{1}^{H}, \Sigma_{2}^{H}, \in\right\rangle$ of Minkowski geometry is isomorphic to the projective model of Minkowski geometry in a projective plane over a Euclidean field.

REMARK 2.1. It follows from Theorem 1.1 that to horocycles, nondegenerate equidistant curves and hyperbolic circles of oriented lines correspond conics of oriented lines in the projective model. A degenerate circle and an equidistant curve are pencils of oriented lines respectively proper and ultraparallel.

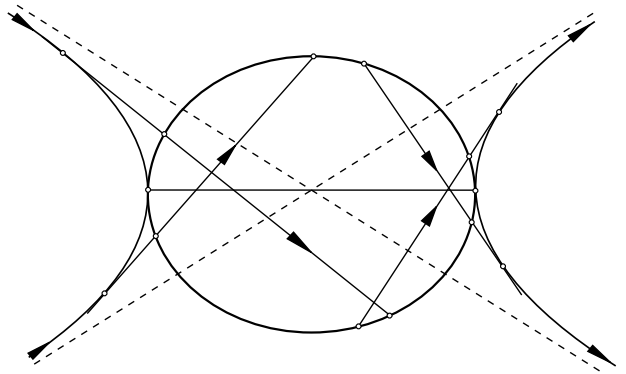

Fig. 2

REMARK 2.2. Our definition of equidistant curve of oriented lines contains a case which has no dual description as orbits of points of the hyperbolic plane. Such an equidistant curve may be obtained as the orbit of the oriented line intersecting the axis of the pencil. The dual curve in the projective model is an oriented conic which does not contain points inside $C$ (Fig. 2). 


\section{Hyperbolic geometry in a bundle of circles of Minkowski}

plane. In this section we show that the geometry in a bundle of circles of any symmetric Minkowski plane is an extended hyperbolic geometry.

Theorem 3.1. Let $\mathcal{M}=\left\langle\mathcal{P}, \Lambda, \Sigma_{1}, \Sigma_{2}, \in\right\rangle$ be a symmetric Minkowski plane with char $\mathcal{M} \neq 2$ and $E$ a fixed circle. Then the structure $\left\langle\mathcal{P}^{E}, \mathcal{L}^{E}, \in^{E}\right.$, $\left.\perp_{h}^{E}\right\rangle$ is an extended hyperbolic plane, where

$$
\begin{gathered}
\mathcal{P}^{E}=E^{\perp} \cup\{p \mid p \in E\}, \quad \mathcal{L}^{E}=\left\{(E, M)^{\perp} \mid M \in \Lambda\right\}, \\
K \in^{E}(E, M)^{\perp} \Leftrightarrow K \in(E, M)^{\perp}, \quad p \in^{E}(E, M)^{\perp} \Leftrightarrow\{p\}=E \cap M, \\
(E, M)^{\perp} \perp_{h}^{E}(E, N)^{\perp} \Leftrightarrow\left\{\left((E, M)^{\perp}=(E, N)^{\perp} \wedge \overline{\overline{E \cap M}}=1\right) \vee\right. \\
\left.\left(\exists M^{\prime}, N^{\prime}\left(M^{\prime} \in(E, M)^{\perp} \wedge N^{\prime} \in(E, N)^{\perp} \wedge M^{\prime}, N^{\prime} \perp E \wedge M^{\prime} \perp N^{\prime}\right)\right)\right\} .
\end{gathered}
$$

Proof. Let us fix the isomorphism $\Phi_{\mathcal{M}}^{E}$. Then to circles of the bundle $E^{\perp}$ correspond circles $K_{\alpha}$ where $\alpha$ is an involution. To any such circle we assign a point $o_{\alpha}$ (the center of $\alpha$ ). To any point $(x, x)$ of the projective model we assign the point $x \in C$. Such a correspondence is a bijection of $\mathcal{P}^{E}$ with the set of points of the projective plane $\mathcal{P}$, because every point of $\mathcal{P} \backslash C$ is the center of a uniquely defined involution of $C$. To every pencil $(E, M)^{\perp}$ represented by $\left(K_{\mathrm{id}}, K_{\beta}\right)^{\perp}$ in a projective model we assign the axis $O_{\beta}$. This definition according to Proposition 1.3 is independent of the choice of the circle $M$ and yields a bijection. We have $K \in^{E}(E, M)^{\perp} \Leftrightarrow o_{\alpha} \in O_{\beta}$, where $\Phi_{\mathcal{M}}^{E}(K)=K_{\alpha}, \Phi_{\mathcal{M}}^{E}(M)=K_{\beta}$ by Proposition 1.3. Moreover $p \in^{E}(E, M)^{\perp}$ exactly when $x$ is the only fixed point of the projectivity $\beta$, where $(x, x)$ corresponds to $p$ in the projective model. This means that $O_{\beta}$ is tangent at the point $(x, x)$ to the absolute $C$. If $M$ is tangent to $E$, then $\Phi_{\mathcal{M}}^{E}=K_{\beta}$ where $\beta$ is a projectivity with unique fixed point. It follows that $O_{\beta}$ is an isotropic line of the extended hyperbolic plane.

If $(E, M)^{\perp} \neq(E, N)^{\perp}$ then by Theorem 1.2 there exist circles $M^{\prime}, N^{\prime}$ such that $M^{\prime} \in(E, M)^{\perp}, N^{\prime} \in(E, N)^{\perp}$ and $M^{\prime}, N^{\prime} \perp E$. Setting $\Phi_{\mathcal{M}^{\prime}}^{E}(K)=$ $K_{\alpha^{\prime}}, \Phi_{\mathcal{M}}^{E}(M)=K_{\beta^{\prime}}$ we find that hyperbolic orthogonality of the lines $O_{\beta^{\prime}}$ and $O_{\gamma^{\prime}}$ is equivalent to orthogonality of the circles $M^{\prime}$ and $N^{\prime}$ in the Minkowski plane (the involutions $\beta^{\prime}$ and $\gamma^{\prime}$ are conjugate).

The above construction of a hyperbolic geometry is complicated and nonhomogeneous. In the case of a Euclidean field the construction becomes simpler and it leads to the classical Klein model.

Corollary 3.1. For any fixed circle $E$ of a symmetric Minkowski plane over a Euclidean field the structure $\left\langle\mathcal{U}^{E}, \mathcal{L}_{U}^{E}, \in, \perp_{U}^{E}\right\rangle$ is a hyperbolic plane, where

$$
\begin{gathered}
\mathcal{U}^{E}=\left\{K \in E^{\perp} \mid K \cap E=\emptyset\right\}, \quad \mathcal{L}_{U}^{E}=\left\{(E, M)^{\perp} \cap \mathcal{U}^{E} \mid M \in E^{\perp}, \overline{\overline{E \cap M}}=2\right\}, \\
(E, M)^{\perp} \cap \mathcal{U}^{E} \perp_{U}^{E}(E, N)^{\perp} \cap \mathcal{U}^{E} \Leftrightarrow M \perp N .
\end{gathered}
$$


Proof. In the isomorphism used in the proof of Theorem 2.1, to circles of the set $E^{\perp}$ which are disjoint from $E$ there correspond centers of involutions without fixed points. For any projective plane over a Euclidean field this is equivalent to the statement that the center of the involution is inside the conic. Because by definition there is no parabolic pencil in $\mathcal{L}_{U}^{E}$, by Proposition 1.2 any pencil $(E, M)^{\perp}$ can be represented by a circle orthogonal to $E$. This also leads to simplification of the condition defining orthogonality.

A synthetic description of Minkowski planes over Euclidean fields will be presented in another paper.

\section{References}

[1] F. Bachman, Aufbau der Geometrie aus dem Spiegelungsbegriff, Springer, Berlin, 1973.

[2] H. Beck, Ein Seitenstück zur Moebius'schen Geometrie der Kreisverwandtschaften, Trans. Amer. Math. Soc. 11 (1910), 414-448.

[3] W. Benz, Vorlesungen über Geometrie der Algebren, Springer, Berlin, 1973.

[4] H. S. M. Coxeter, Non-Euclidean Geometry, Math. Expositions 2, Univ. of Toronto Press, Toronto, 1978.

[5] J. Ch. Fischer, Models and theorems of the classical circle planes, Abh. Math. Sem. Univ. Hamburg 63 (1993), 245-264.

[6] W. Heise und H. Karzel, Symmetrische Minkowski-Ebenen, J. Geometry 3 (1973), $5-20$.

[7] H. Karzel, Symmetrische Permutationsmengen, Aequationes Math. 17 (1978), 8390 .

[8] J. Kosiorek and A. Matraś, The model of the Minkowski plane in the projective plane with a fixed conic, submitted to Algebra Geom. Appl.

[9] M. Kordos, The Foundations of the Projective and Projective-Metric Geometry, PWN, Warszawa, 1984 (in Polish).

[10] H. Mäurer, Laguerre- und Blaschke-Modell der ebenen Laguerre-Geometrie, Math. Ann. 164 (1966), 124-132.

[11] O. Veblen and J. W. Young, Projective Geometry, Vol. 1, Boston, 1910.

[12] H. Zeitler, On reflections in Minkowski planes, in: Lecture Notes in Math. 792, Springer, 1980, 183-201.

Jarosław Kosiorek and Andrzej Matraś

Faculty of Mathematics and Computer Science

University of Warmia and Mazury

Żołnierska 14

10-561 Olsztyn, Poland

E-mail: matras@uwm.edu.pl

Received March 1, 2004;

received in final form April 16, 2004 